Published as: Vanclooster, A., Ooms, K., Viaene, P., Fack, V., Van de Weghe, N., De Maeyer, P. (2014), Evaluating suitability of the least risk path algorithm to support cognitive wayfinding in indoor spaces: an empirical study. Applied Geography, vol. 53: 128-140.

\title{
Evaluating suitability of the least risk path algorithm to support cognitive wayfinding in indoor spaces: an empirical study
}

Ann Vancloostera, Kristien Oomsa, Pepijn Viaenea, Veerle Fack ${ }^{b}$, Nico Van de Weghea, Philippe De Maeyera

a Department of Geography - Ghent University

Krijgslaan 281 (S8)

B-9000 Ghent

Belgium

b Department of Applied Mathematics and Computer Science - Ghent University

Krijgslaan 281 (S9)

B-9000 Ghent

Belgium

\section{E-mail addresses:}

\{Ann.Vanclooster; Kristien.Ooms; Pepijn.Viaene; Veerle.Fack; Nico.VandeWeghe;

Philippe.DeMaeyer\}@Ugent.be

\section{Corresponding author:}

Ann Vanclooster

E-mail: Ann.Vanclooster@Ugent.be

Tel: 003292644727

Fax: 003292644985 


\title{
Evaluating suitability of the least risk path algorithm to support cognitive wayfinding in indoor spaces: an empirical study
}

\begin{abstract}
Over the last couple of years, applications that support wayfinding in indoor spaces have become a booming industry. Finding one's way in complex 3D indoor environments can still be a challenging endeavor, partly induced by the specific indoor structure (e.g. fragmentation, less visibility, confined areas). Appropriate algorithms that help guide unfamiliar users by providing 'easier to follow' route instructions are so far mostly absent indoors. In outdoor space, several alternative algorithms exist, adding a more cognitive notion to the calculated paths and as such adhering to the natural wayfinding behavior (e.g. simplest paths, least risk paths). The aim of this research is to extend those richer cognitive algorithms to three-dimensional indoor environments. More specifically, the focal point of this paper is the application of the least risk path algorithm, i.e. an algorithm developed to minimize the risk of getting lost, to an indoor space. This algorithm is duplicated and extensively tested in a complex multi-story building by comparing the quality of the calculated least risk paths with their shortest path alternatives. The outcome of those tests reveals non-stable results in terms of selecting the least risky edges in indoor environments, which leads to the conclusion that the algorithm has to be adjusted to the specificities of indoor space. Several improvements for the algorithm are proposed and will be implemented as part of future work to improve the overall user experience during navigation in indoor environments.
\end{abstract}

\section{Keywords}

Indoor, Navigation, Algorithm, Wayfinding

\section{Introduction}

Finding one's way in unfamiliar environments can sometimes turn out to be a challenging endeavor as people get disoriented and lose their way. Golledge (1999) defines being lost as 'a state which occurs when the wayfinding process fails in some way'. In behavioral and cognitive sciences, navigation processes have already been widely studied (both indoor and outdoor) with navigation typically defined as cognitively consisting of locomotion and wayfinding components (Montello, 2005). Wayfinding is thereby the process of determining and following a route between origin and destination and is often guided by external aids (Golledge, 1999). In the context of this paper, we focus on these guidance aids that can improve wayfinding and not on the cognitive act of wayfinding itself.

The setting for our research is limited to indoor spaces as wayfinding research in indoor environments has repeatedly demonstrated the challenges of successfully performing navigation tasks in a complex three-dimensional space (e.g. disorientation after vertical travel, less visual routing aid, deficient cognitive map creation) (Hölscher et al., 2009). Appropriate guidance to simplify the act of wayfinding is hereby a crucial factor, especially for unfamiliar users that will rely more heavily on external indoor navigation aids. Such navigation aids come in various forms, but all contain some kind of model of space enhanced with routing instructions and localization technology (Nagel et al., 2010). In the last decade, a wide variety of indoor navigational models (Brown et al., 2013) have been developed, but a general framework still has 
to reach full maturity (Nagel et al., 2010). Apart from these typical network models based on traditional graph theory, the Space Syntax society opened up research on aspects of visibility and connectivity in spatial building configurations and their impact on pedestrian movement (e.g. Parvin et al., 2007; Turner et al., 2001). These models will however not be considered in the current research.

Beside navigational models, navigation guidance also relies on appropriate and accurate algorithmic support. Algorithms for 3D indoor navigation are currently restricted to Dijkstra (1959) or derived shortest path algorithms (e.g. Kwan \& Lee, 2005; Thill et al., 2011). However, the results of those algorithms often exhibit non-realistic paths (e.g. using complex intersections, avoiding main walking areas) in terms of what an unfamiliar indoor wayfinder would need, to navigate a building comfortably. To date, few researchers have attempted to approach algorithms for indoor routing differently, for example incorporating dynamic events (Musliman et al., 2008), or modelling evacuation situations (Atila et al., 2013; Vanclooster et al., 2012). In contrast, for outdoor environments, several 'cognitive' algorithms (e.g. paths minimizing route complexity (Duckham \& Kulik, 2003; Richter \& Duckham, 2008), hierarchical paths (Fu et al., 2006)) have been developed that add a more qualitative description to routes by using a more cognitive cost heuristic than traditional shortest path algorithms (Table 1).

Table 1: Comparison of several cognitive algorithms and their cost heuristic

\begin{tabular}{ll}
\hline Algorithm & Cost heuristic (minimization criterion) \\
\hline Shortest path algorithm (e.g. Dijkstra, 1959) & Path length \\
Hierarchical shortest path algorithm (Fu et al., 2006) & Computational time \\
Simplest path algorithm (Duckham \& Kulik, 2003) & Intersection complexity (number of edges + intersection \\
& type) \\
Simplest path algorithm (Mark, 1986) & Path length + intersection complexity \\
Simplest instruction algorithm (Richter \& Duckham, & Intersection complexity + spatial chunking \\
2008) & \\
Least risk path algorithm (Grum, 2005) & Path length (50\%) + Risk value (50\%) \\
\hline
\end{tabular}

These 'cognitive' algorithms have the aim to simplify wayfinding by providing routes that are easier to follow, more intuitively correct, and in general more adhering to how people conceptualize routes to unfamiliar users (Tsetsos et al., 2006). Several cognitive studies have indeed indicated that during routing, humans value equally as much the form and complexity of route instructions as the total path length (Duckham \& Kulik, 2003). These algorithms have not yet been implemented in indoor cases, although the need for cognitively rich algorithms is even more pronounced in indoor space compared to outdoors. As such, the main goal of our research is to translate existing outdoor 'cognitive' algorithms to an indoor environment and provide indoor route calculations that are more aligned with indoor wayfinding behavior. In a different part of our study, the implementation of the simplest path algorithm in indoor environments is being considered.

However, this paper explicitly focuses on the implementation and testing of the least risk algorithm of Grum (2005) in a three-dimensional indoor environment. The least risk path algorithm, minimizing the risk of getting lost, is especially interesting for indoor application as the structure of indoor spaces induces more getting-lost episodes (Hölscher et al., 2006). An algorithm lowering the probability of getting lost by taking less complex paths could as such 
prove valuable in reducing indoor wayfinding difficulties. Specifically, we want to investigate whether the results of the least risk path algorithm have the same connotation and importance in indoor spaces as in its original outdoor setting. Also, the least risk path algorithm is analyzed for its applicability in providing route instructions that adhere better to the natural wayfinding behavior of unfamiliar users in indoor space.

The remainder of the paper is organized as follows: section 2 elaborates on the definition of risk in the algorithm and for indoor wayfinding; in section 3, a case study is presented to evaluate the algorithm for its suitability in supporting indoor cognitive routing; section 4 discusses the conclusions from our study and possible improvements for the algorithm.

\section{Defining the risk of getting lost in indoor wayfinding}

\subsection{Least risk path algorithm}

The least risk path algorithm as described by Grum (2005) calculates the path between two points where a wayfinder has the least risk of getting lost by selecting all edges and intersections with a minimal risk value. This risk value is measured at every intersection and is defined by the cost for taking a wrong decision at that intersection. The algorithm assumes that (1) the person taking the path is unfamiliar with its environment, and (2) when taking a wrong path segment, the wayfinder notices this immediately and turns back at the next intersection (Grum, 2005). While these assumptions might be quite strict, Grum (2005) also acknowledges that the algorithm needs to be tested for its representativeness of the actual behavior of users.

The formula for the calculation of the risk value at intersection $i$ and the total risk of an entire path $p$ is as follows:

$$
\begin{aligned}
& \text { Total_Risk }(p)=\sum \text { PathLengths }+\sum \text { RiskValue }(i) \\
& \text { RiskValue }(i)=\frac{2 * \sum \text { PathLength_Wrong_Choices }}{\text { No_Possible_Choices }}
\end{aligned}
$$

Equation 2 indicates that the risk value is dependent on the number of edges converging on the decision point, combined with the length of each individual segment and is as such a measure of average length of a wrong edge at that intersection. The multiplication by two points at the idea that, when taking a wrong edge, the user is supposed to return immediately along the same edge, traversing that edge twice. By defining the risk value in this way, the algorithm favors paths with combined long edges and easy intersections. The formula for the total risk of a path (Equation 1) balances the sum of all intersection-based risk values with the length of the actually taken edges. Both elements contribute in this case equally to the total risk of a certain path.

The algorithmic structure of the least risk path algorithm is similar to Dijkstra's shortest path algorithm with a continuous loop over all nodes including the following three consecutive steps:

1 Detect the next smallest node

2 Change the selected node to the next smallest node

3 Adjust cost calculation for adjacent nodes (Fig. 1\&2)

However, in the third step, the least risk path differs significantly from the Dijkstra algorithm since the cost value is not only dependent on the length of the edge but also on the risk value of 
each intersection that is passed which in turn is dependent on the previous route taken and the length of its adjacent edges. The following steps in the 'adjust cost calculation' are consecutively executed:

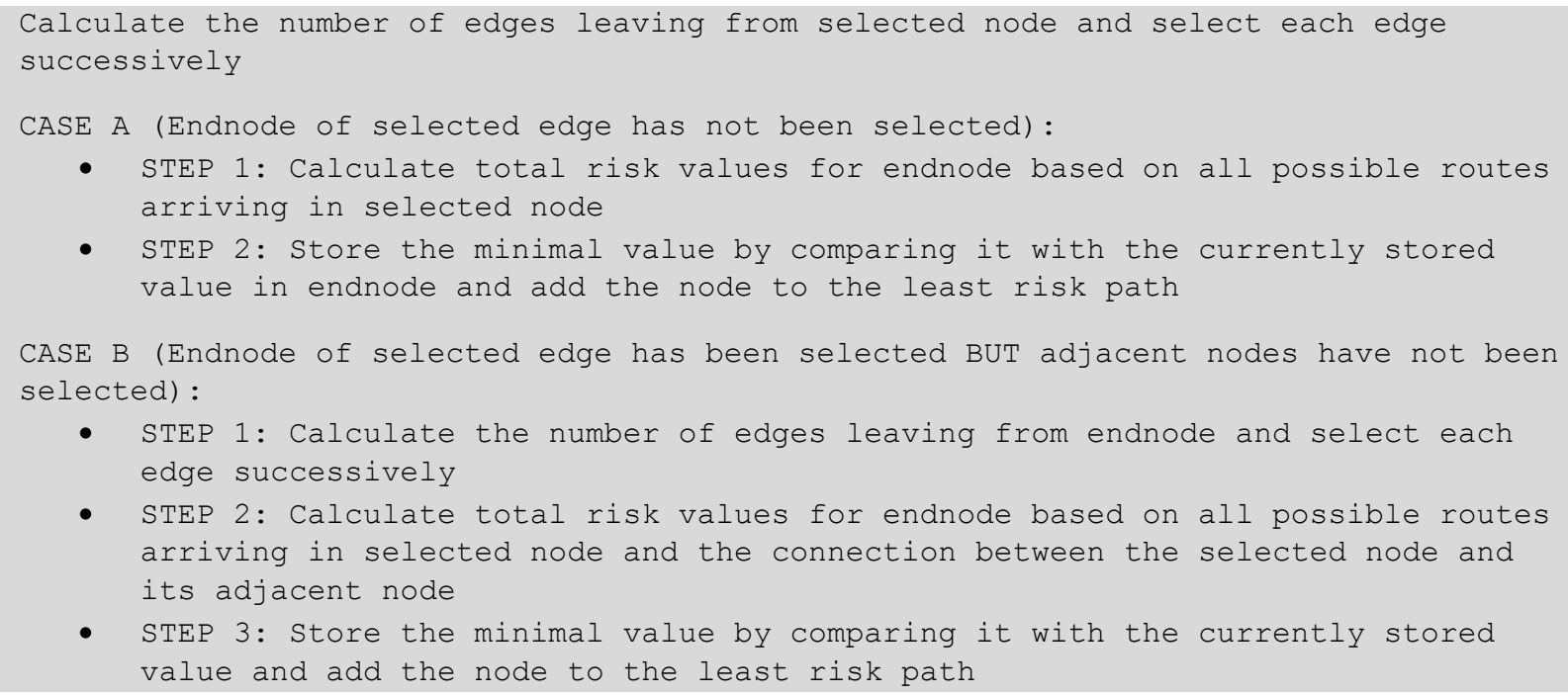

Fig. 1: Algorithm for the adjust cost calculation for adjacent nodes

Fig. 2 shows an example network with two consecutive situations during the execution of the 'adjust cost calculation'. Fig. 2 (left) illustrates the case where Node4 is selected as next smallest node in the network. Node4 has a least risk path of [Node0-Node2-Node4]. From Node4 all edges leaving this node (i.e. edges $a, b, c)$ are consecutively chosen and new total risk values are calculated for their respective endnodes (i.e. Node3, 5, 6). To calculate the total risk value for Node5 with path [Node0-Node2-Node4-Node5], the risk value of Node4 together with path length $\mathrm{b}$ is added to the total risk value of Node4. Node5 and Node6 are in this case calculated for the first time (Case a). Node3 has been calculated before with path [Node0-Node1-Node3]. These previous total risk values are compared with the newly calculated values for the path [Node0-Node2-Node4-Node3] and only those values are stored that are the smallest in total cost (Case a).
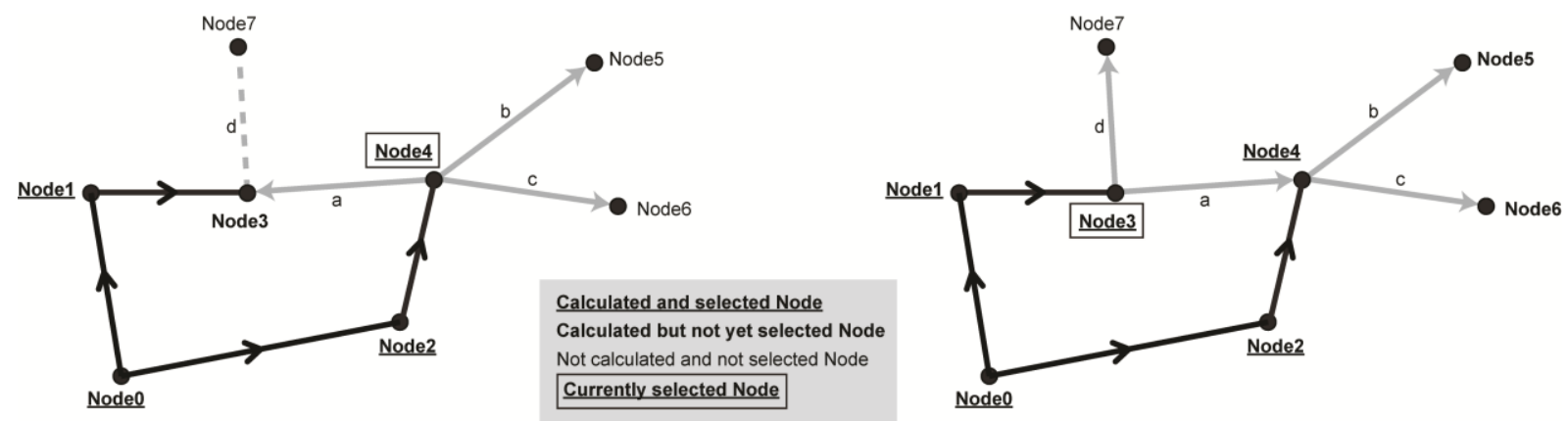

Fig. 2: Two example situations of the implementation of the adjust cost calculations algorithm for adjacent nodes

Fig. 2 (right) illustrates the next situation in the algorithm. From all nodes being calculated but not yet selected (i.e. Node3, 5, 6), Node3 has the smallest cost values and is the next selected node. His least risk path is hereby defined as [Node0-Node1-Node3]. Again, all neighboring edges $(a, d)$ and endnodes (Node7, 4) are chosen. Node7 has not yet been selected nor calculated (case a) and will be calculated as a path [Node0-Node1-Node3-Node7]. As Node4 has already 
been calculated and selected (Case b), Node5 and Node6 are being calculated with previous pathnodes [Node0-Node1-Node3-Node4] as this path could possibly be less costly than through (the already saved cost of) path [Node0-Node2-Node4]. The total risk values for both possibilities are compared in case $b$ and the smallest value is stored.

Given the fact that the only difference with the Dijkstra algorithm is in the cost calculation, and there the additional calculations only affect the amount of edges in the selected node, the computational complexity is similar to Dijkstra, being $\mathrm{O}\left(n^{2}\right)$.

\subsection{Theoretical definition of the risk of getting lost in wayfinding research}

As defined in the previous section, the goal of the least risk path algorithm is to minimize the risk of getting lost. However, Grum's algorithm does not clearly state what a 'minimal' risk exactly signifies, especially given the complexity of indoor wayfinding for unfamiliar users. Several methodologies can be suggested to determine the actual riskiness of paths, ranging from physically testing the accurateness with real test persons, to simulating the wayfinding problems in an agent-based environment. For this paper, as a benchmark we selected a series of objective parameters that have been demonstrated, in previous wayfinding literature, to contribute to the risk of getting lost in both indoor and outdoor space.

It is believed that three factors contribute to the ease of getting lost in buildings during wayfinding: the spatial structure of buildings, cognitive maps created during wayfinding and the individual strategies and spatial abilities of the user (Carlson et al., 2010; Hölscher et al., 2006). At this point, we only account for the structure of the building itself for several reasons. First, Hölscher et al. (2006, p. 284) specifically state: 'many have wayfinding problems because of architecture that only rudimentarily accounts for human spatial cognition'. Peponis, et al. (1990) agree that the degree of wayfinding is mainly dependent on configurational factors. Second, an algorithm that supports wayfinding in various building settings and for various user typologies should be independent of specific spatial-cognitive abilities of a certain user. Also, not all users of a building are at the same level in terms of ability, strategy selection or experience (Carlson et al., 2010). Third, the algorithm is developed for aiding unfamiliar users in their wayfinding tasks. The users therefore have not yet built up a cognitive map of the environment. As such, the parameters, proposed as benchmark, define the theoretical risk of getting lost during wayfinding and all relate specifically to the spatial building structure itself (Table 2).

Table 2: Benchmark parameter set and their significance for wayfinding

\begin{tabular}{ll}
\hline Benchmark parameter & Significance for wayfinding \\
\hline Route efficiency & Total path length (Hölscher et al., 2011) \\
Route complexity & $\begin{array}{l}\text { Number of turns and streets used (Hölscher et al., 2011), also referred to as step } \\
\text { depth (Hölscher et al., 2006) } \\
\text { Number of curves }\end{array}$ \\
In wayfinding, the direction strategy, often used by familiar users, continuously \\
minimizes the angle between destination and current position (Hölscher et al., 2011). \\
Less curves help following this strategy and maintain indoor orientation. Unfamiliar \\
users, following a planned strategy, also benefit from fewer curves to feel more at \\
ease and keep orientation. \\
Wide streets are considered more salient (Hölscher et al., 2011). Equivalent in indoor \\
space, the selection of wider corridors can be important to reduce the risk of getting \\
lost.
\end{tabular}


Redundancy

Integration value

Probability of path choice at an intersection

Number of visible decision points
I.e. a decrease in decision points that the user has to pass. Fewer nodes along a path have proven to decrease wayfinding difficulties (Peponis et al., 1990).

Quantifies to what extent each space is directly or indirectly connected to other spaces. People naturally move to the most integrated nodes when navigating through a building (Peponis et al., 1990). Novices rely even more on following the paths of high connectivity and integration (Hölscher et al., 2012).

I.e. the weighting of which paths are most likely to be taken. An uneven distribution of probability exists at each intersection, especially given the fact that more integrative spaces naturally gather more people (Peponis et al., 1990).

Unfamiliar participants, during the initial exploration of a building, rely mostly on local topological qualities, such as how many additional decision points could be seen from a given node (Haq \& Zimring, 2003). Also, a lack of survey places with open views and long lines of sights has shown to enhance stops and hesitations (Hölscher et al., 2012). Apparent dead ends often lead to misunderstanding and make people less reluctant to choose this path (Hölscher et al., 2012).

These parameters (Table 2) all influence the chances of getting lost during wayfinding and will help determine whether the proposed least risk paths coincide with theoretically defined parameters of riskiness. However, the individual weighting of these parameters still has to be decided on. Therefore, we currently use this benchmark set as a way to analyze several example routes that have been calculated (Section 3.3.2). A more elaborate evaluation is planned as future work for adjusting the initial cognitive algorithm.

\section{Case study}

\subsection{Dataset: creation and model}

The applicability of the least risk path algorithm for use in complex indoor environments is evaluated by thoroughly testing it in a case study building. The selected indoor environment is the 'Plateau-Rozier' building of Ghent University. It is a complex multi-story building with several wings and sections, arranged over different floor levels, not all of them being immediately accessible. It is assumed that the mapped indoor space is complex enough with many corners and decision points to assume reasonable wayfinding needs for unfamiliar users. Indeed, previous research executed in this building has shown that unfamiliar users can have considerate difficulty recreating a previously shown route through the building (Viaene \& De Maeyer, 2013).

For application of the least risk path algorithm, the original floor plans have been manually converted into a three-dimensional indoor network structure (Fig. 3). Automatic derivation of indoor networks has long been focused on as one of the problematic areas for indoor navigation applications. Recent efforts have shown possibilities of automatically assigning nodes to each room object and connecting them when they are connected in reality (Anagnostopoulos et al., 2005; Meijers et al., 2005; Stoffel et al., 2008). However, the development of a comprehensive methodology for automatic network creation requires a thorough foundation and agreement on the appropriate and optimal (i.e. user friendly) network structure of indoor environments which 
supports the user in his navigation task (Becker et al., 2009). Up to this point and as far as we know, this is still missing in indoor navigation research.
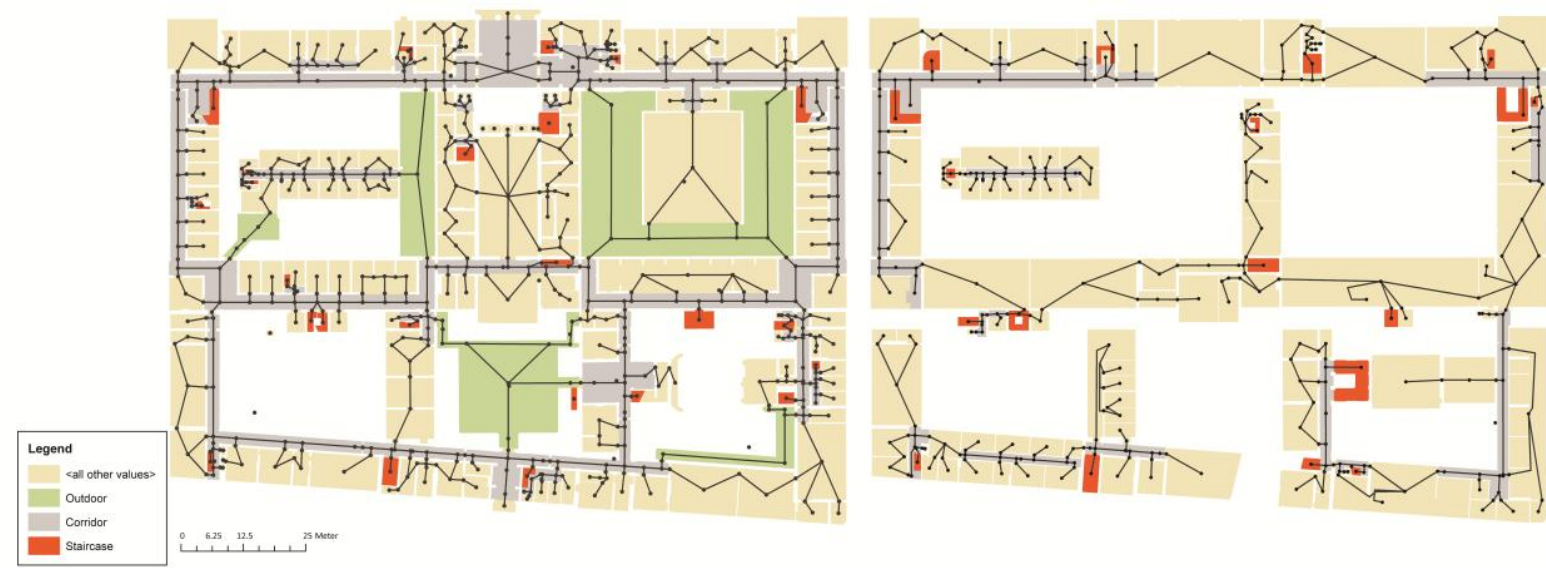

\section{Fig.3: Floor plan of the ground floor (left) and first floor (right) with their 3D indoor network.}

For this research, only the ground floor and first floor were considered. The network structure is chosen to be compliant to Lee's Geometric Network Model (Lee, 2004) as this structure is widely accepted and is currently put forward as indoor network model in the IndoorGML standard proposal (OGC, 2013). In this model, each room is transformed into a node, forming a topologically sound connectivity model. Afterwards, this network is transformed into a geometric model by creating a subgraph for linear phenomena (e.g. corridors), which enables network analysis. The position of the node within the rooms is selected to be the geometrical center point of the polygons defining the rooms. This premise implies that the actual walking pattern will sometimes not be conform to the connectivity relationships in the network inducing small errors in the calculations of shortest and least risk paths. We will need to verify whether or not this error is significant in the total cost of certain paths. The selection of corridors to be transformed into linear features is based on the map text labels indicating corridor functionality. These areas also appear to be perceived as corridors when inspecting the building structure itself in the field. Obviously, this topic is depending on personal interpretation and choice. Therefore, in future research, the dependency of the performance of cognitive algorithms on the indoor network topology will be investigated.

\subsection{General results of analysis}

The goal of this case study is to assess the least risk path algorithm for use in indoor environments and this by comparing the calculated paths of the least risk path algorithm with the results of the shortest path algorithm. More specifically, we want to (1) compare how much the least risk paths decrease the risk of getting lost compared to the shortest paths, (2) if the least risk path algorithm actually reduces the navigational complexity of the paths and (3) if the results of the least risk path calculations indoor have a similar improvement to their shortest path equivalents compared to the outdoor case.

The entire dataset of the case study building consists of more than 600 nodes and more than 1300 edges. This required a computation of almost 800,000 paths to exhaustively calculate all possible paths between all nodes for both the shortest path and least risk path algorithm. This will also include trivial paths (e.g. between close neighbors) without any path difference. However, we chose to compare all paths instead of defining an arbitrary distance without any 
theoretical foundation. For each path, the total length and risk values for the intermediate nodes are calculated in both the shortest and least risk path algorithm.

\subsubsection{Path length and risk value comparison}

Over the entire set of results, on average the difference in path length between least risk paths and their respective shortest paths is found to be around $4.5 \mathrm{~m}$ with a decrease in risk value of $15.6 \mathrm{~m}$ (i.e. the average sum of the lengths of wrong edges at each intersection along the path). These values align with the original definition of both algorithms and their different cost minimization criterion. The length of a path described by the least risk path algorithm (total risk value minimization) is designed to be equal or longer than its equivalent shortest path (length minimization) by providing a less risky detour. The least risk path algorithm will more likely calculate routes with fewer intersections, away from the major corridors where many choices appear, while the shortest path will go for the most direct option ignoring the complexity of the individual intersections.

Over the entire dataset, a least risk path indoor is on average $4 \%$ longer than its respective shortest path. Although $53 \%$ of least risk paths are longer than their equivalent shortest paths, the majority (almost 99\%) of paths are less than a quarter longer (Table 3). This indicates that while half of all paths seem to deviate from the shortest path to obtain a theoretically less risky route (otherwise their lengths would be equal), those deviations are mostly limited in size. Taking into consideration that the total path length of both shortest and least risk paths in this indoor space are already quite short (109.42 m to $113.89 \mathrm{~m}$ with standard deviations of $45.69 \mathrm{~m}$ and $48.54 \mathrm{~m}$ respectively) due to the restricted building size, the found limited path length differences are of even less significance. Most deviations from the shortest path will only have a single node-edge couple difference. These results point to an at first sight almost equivalent path choice by both algorithms, implying that either (1) the shortest path algorithm is already selecting paths that are least risky to get lost on or, (2) they give an indication that the least risk path algorithm is actually not calculating less risky routes and as such might not be well defined for use in indoor spaces. A further examination of both ideas follows in sections 3.2.2 and 3.3.

Table 3: Classification of path length increase

\begin{tabular}{lrc}
\hline \multicolumn{1}{c}{ Length increase } & Number of paths & Ratio of total paths \\
\hline Equal path lengths & 160,984 & 46.64 \\
]0\%-5\%] & 87,681 & 25.40 \\
]5\%-10\%] & 50,773 & 14.71 \\
]10\%-25\%] & 41,196 & 11.94 \\
]25\%-50\%] & 4,363 & 1.26 \\
$>50 \%$ & 159 & 0.05 \\
\hline TOTAL & 345,156 & 100.00 \\
\hline
\end{tabular}

\subsubsection{Navigational complexity analysis}

As the aim of the least risk path algorithm is to lower the total risk of getting lost, the type of selected paths and more specifically their navigational complexity should be lowered given an increased total path length. Navigational complexity is in this case defined by the number of intersections passed and the average number of choices at intersections. Table 4 shows that for both the number of intersections and the average number of choices at an intersection the results are lower in the case of the least risk path algorithm than for the shortest path algorithm. 
However, the differences are quite small which demonstrates that the least risk path algorithm does not significantly decrease the navigational complexity of the final path.

Table 4: Summary of the navigational complexity results over the entire dataset

\begin{tabular}{lcr}
\hline & Shortest Path algorithm & Least Risk Path algorithm \\
\hline Number of intersections & 18.16 & 17.84 \\
Average number of choices at an intersection & 3.09 & 3.03 \\
\hline
\end{tabular}

A classification of the paths according to length increase (Table 5) shows (1) that for both risk value and average number of choices the values gradually decrease for least risk paths with increasing path length differences. These results are as expected as for having a significant deviation from the shortest path, the least risk path should provide in avoiding significantly riskier areas to get lost than the alternative paths. Although even with less complex intersections for the least risk path algorithm, the differences are still almost negligible. Remarkably, (2) for the number of intersections, least risk paths with large increases in total path length show an increase in number of intersections compared to the shortest paths. As the initial point of the algorithm is to lower the total risk of getting lost as a whole, even with a path length increase it should contain fewer and less complex intersections. This is at this point not the case for the number of intersections. Again, all differences appear to be quite small, validating the originally raised questions about the applicability of the original least risk path definition for indoor usage.

Table 5: Differences following the classification in path length increase

\begin{tabular}{lccc}
\hline \multicolumn{1}{c}{ Length increase } & $\begin{array}{c}\text { \% increase in Risk } \\
\text { Value }\end{array}$ & $\begin{array}{c}\text { \% increase in number of } \\
\text { intersections }\end{array}$ & $\begin{array}{c}\text { \% increase in average } \\
\text { number of choices }\end{array}$ \\
\hline Equal path lengths & 0.00 & 0.00 & 0.00 \\
] $0 \%-5 \%]$ & -0.18 & -0.04 & -0.02 \\
] $5 \%-10 \%]$ & -0.34 & -0.04 & -0.04 \\
] $10 \%-25 \%]$ & -0.51 & -0.02 & -0.06 \\
] $25 \%-50 \%]$ & -0.70 & 0.05 & -0.09 \\
$>50 \%$ & -1.05 & 0.08 & -0.21 \\
\hline
\end{tabular}

\subsubsection{Comparison with the outdoor case}

Compared to the results obtained by Grum (2005) in the original outdoor setting, the total risk value for the least risk path is minimal and the length is longer than its shortest path. The outdoor least risk path is $9 \%$ longer than the shortest path, while in our dataset an average increase of $4 \%$ is detected. However, a true comparison between indoor and outdoor results is difficult as the author only calculated a single path in outdoor space. With respect to the results of the navigational complexity, the outdoor least risk path has more intersections (14 versus 12 in the shortest path) but a lower average number of choices at each intersection (3.14 versus 3.5). These results are also in accordance with the findings in the indoor setting, but again these results should be cautiously approached given the limited number of calculations in the outdoor variant.

\subsection{Path embedding in indoor space}

This section focusses on the actual paths themselves and their spatial embedding, i.e. the spatial location of the edges and nodes. More specifically, we will (1) calculate the correlation between shortest and least risk paths and (2) assess the actual riskiness of the paths by relating to the 
previously defined benchmark parameters. The general aim is to identify how alike or different the calculated paths are and if the selected edges are avoiding complex and confusing areas in the building to ensure a lower risk of getting lost.

\subsubsection{Correlation between paths}

For calculating the correlation between each shortest and least risk path, the entire path was rerun with comparisons edge per edge. For a general path correlation measure, an overlap ratio is defined as the sum of all edge lengths that are mutually used in both the least risk and shortest path calculations divided by the total path length of the shortest path. On average, over the entire dataset, an overlap factor of $80 \%$ is found; for the subset of data with paths with different spatial embedding an average overlap of $62 \%$ is found. This result is in both cases quite high, confirming that most paths have a similar spatial embedding between both algorithms. Divided over the various classes of path length increase (Fig. 4), it is obvious that with a large path length increase for the least risk path algorithm, the overlap between shortest and least risk path sharply diminishes as both paths are considerably different in length. With this subset of paths with a path length increase, on average $82 \%$ of intersections on the shortest paths are located in a corridor, while this value is reduced to $78 \%$ for the least risk path algorithm. This demonstrates that when deviations from the shortest path are made, these mostly occur by avoiding main corridor areas.

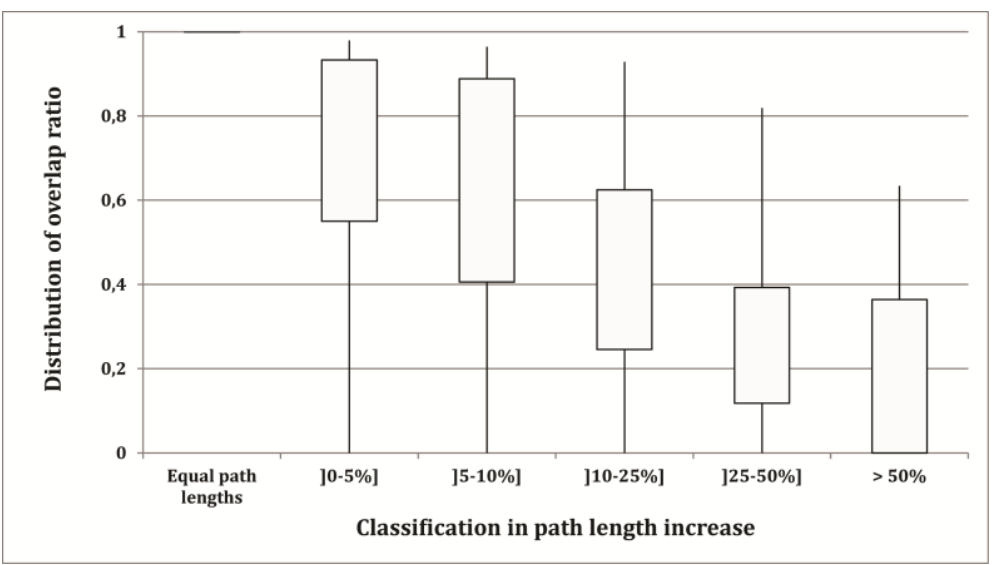

Fig. 4: Distribution of overlap ratio per class of path length increase

A second analysis aims to demonstrate the edge use, defined as the number of times all paths from a certain source node pass by this edge. This analysis was applied to an example source node to maintain visualization clarity, but the calculation is applicable to all source nodes. The result is a map showing the use of each edge by varying line thickness, and this for both the shortest path and least risk path algorithm. The example source node is located in a room in the upper left corner on the first floor, close to a main staircase. Fig. 5 shows a significant difference in the resulting embedding of paths between the shortest path and least risk path algorithm, even though the average path length and risk value difference is respectively limited to $7.7 \mathrm{~m}$ and $13.9 \mathrm{~m}$, which is in line with the found limited differences. More in detail, in the Dijkstra case, from the source node a large amount of paths stay on the first floor to go to a more southern located staircase and deviate from there to the specific rooms. For the least risk path algorithm, to access the same nodes in the southern part of the building on the ground floor, a large amount of paths immediately descend to the ground floors and choose a specific corridor and outdoor area to find their way through the building. Additionally, nodes that have limited path choice generally take the same path in both cases (for example the northeast corner and 
middle/middle-east corridor on $1^{\text {st }}$ floor). Although the conclusions above are specific for this example, these results also imply that the location of the stairs is of major importance in the selection of the paths.

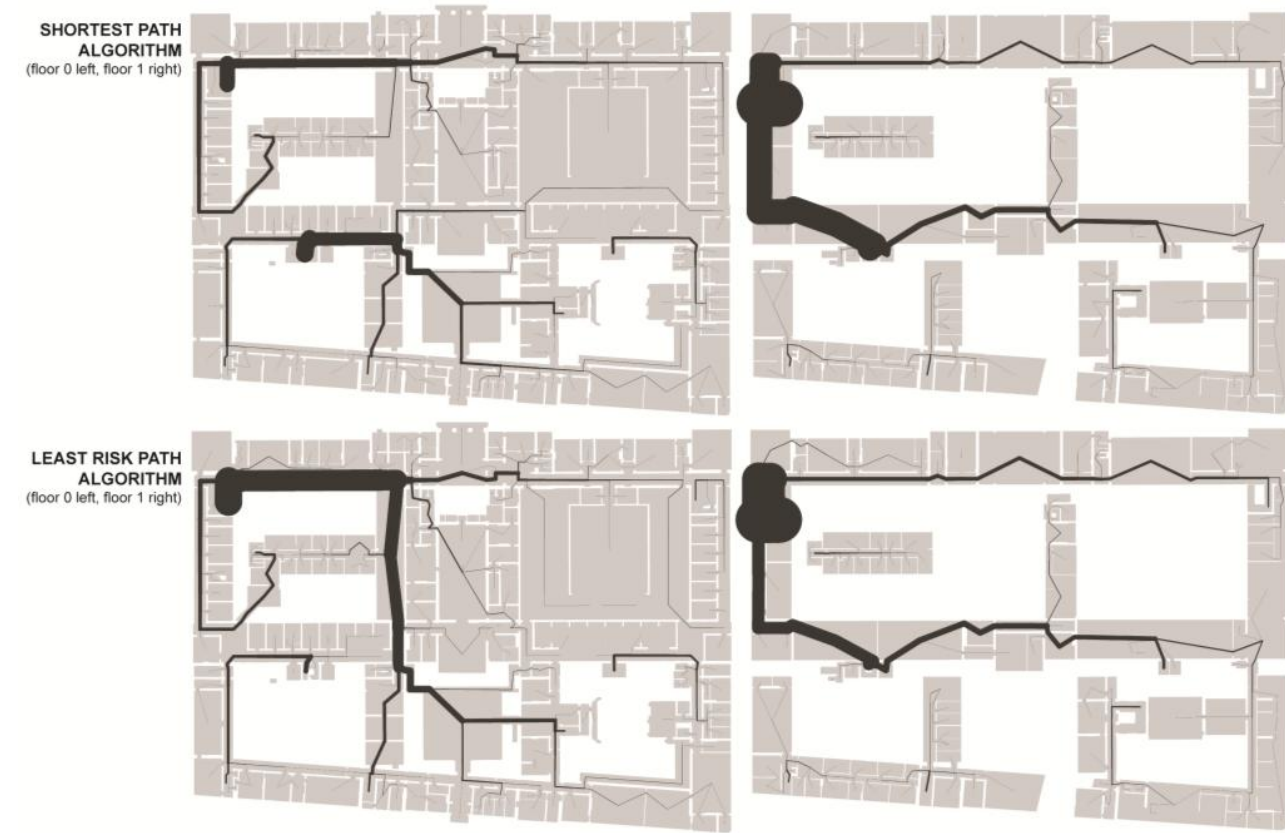

Fig. 5: Path use of the shortest path and least risk path algorithm (source node 1086)

\subsubsection{Benchmark comparison}

In this section we specifically look at the paths which have a different spatial embedding and investigate if the selected least risk path edges in those cases are actually less risky than the ones selected by the shortest path algorithm. The edges are examined on their theoretical riskiness, as defined by the benchmark parameter set, i.e. parameters that have proven to be influencing the risk of getting lost in various wayfinding experiments (Section 2.2).

The first example relates to the analyses in Fig. 5, as it showed significant path embedding differences for certain areas. All paths with start point on the first floor and end point somewhere in the grey rectangle on the ground floor are analyzed. The dashed line in Fig. 6 designates the least risk paths, while the black line visualizes the shortest paths to the grey rectangle.
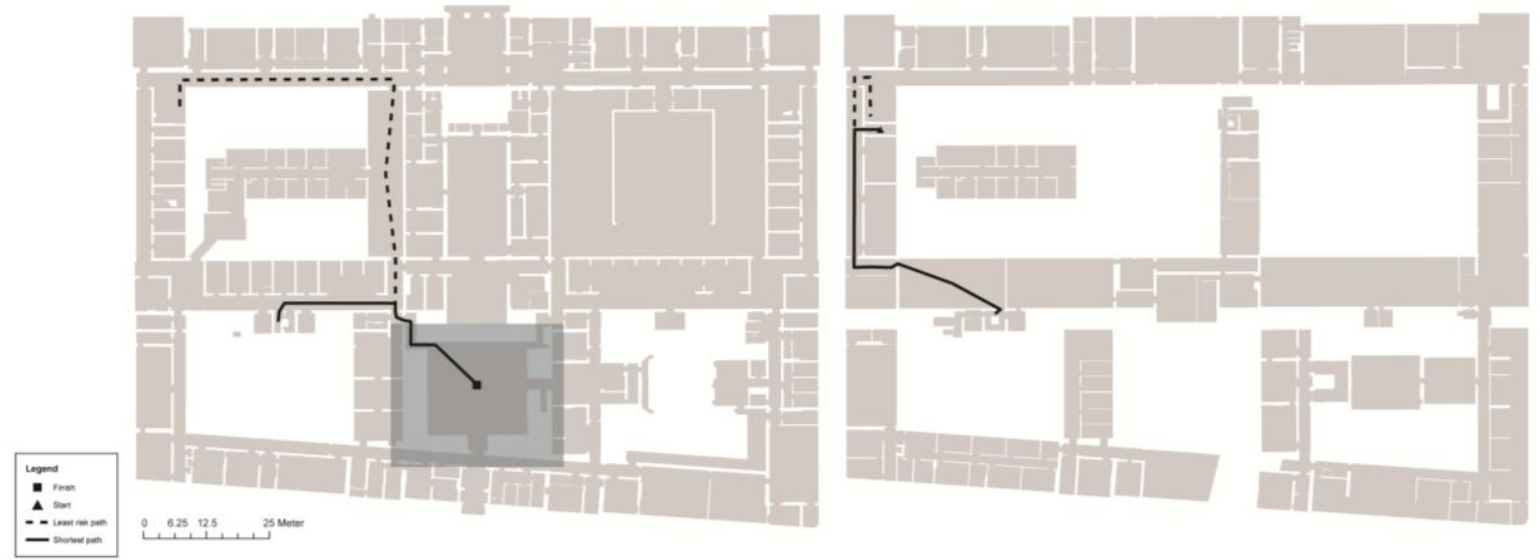

Fig. 6: Path visualization comparing shortest and least risk path (floor 0 left, floor 1 right) 
With respect to the parameters in the algorithm itself, the results in Table 6 show that the least risk paths are significantly less risky (according to its definition) by taking a $21 \%$ longer route (in this example). The other parameters as defined in the benchmark set show quite similar results for both algorithms. The number of turns and curves and the width of corridors are equivalent, as is the number of spatial units passed. Regarding general visibility and lines of sight along the path, the least risk path algorithm shows slightly better results. It can be concluded that both paths are theoretically fairly similar in terms of riskiness. However, in this case, the authors would probably suggest the least risk path as path to an unfamiliar user, mostly because the edges that are selected traverse major corridors and a very visible staircase. The path taken by the shortest path algorithm has to traverse a spatial unit labelled 'room' to reach a minor staircase on the first floor. The other edges being part of the shortest path are equivalent in importance. This example shows that sometimes minor differences determine whether a path is suitable to be recommended for unfamiliar users.

Table 6: Results of the benchmark parameters for the example

\begin{tabular}{lcc}
\hline Benchmark parameters & Shortest paths & Least risk paths \\
\hline Risk value of the entire path $(\mathrm{m})$ & 103 & 67 \\
Total path length & 128 & 155 \\
\hline No. of turns & 9 & 9 \\
No. of spatial units passed & 8 & 1 \\
No. of curves & 1 & 3.2 and 5 \\
Width of corridors $(m)$ & 3.2 & 25 \\
No. of decision nodes passed & 29 & 1.5 \\
No. of visible decision nodes at each decision node (average) & 2 & \\
\hline
\end{tabular}

A second example shows a shortest and least risk path with both start and end points being located on the ground floor (Fig. 7). This example is chosen as it resulted in one of the largest differences in path length increase, and the path choice itself is also significantly different.

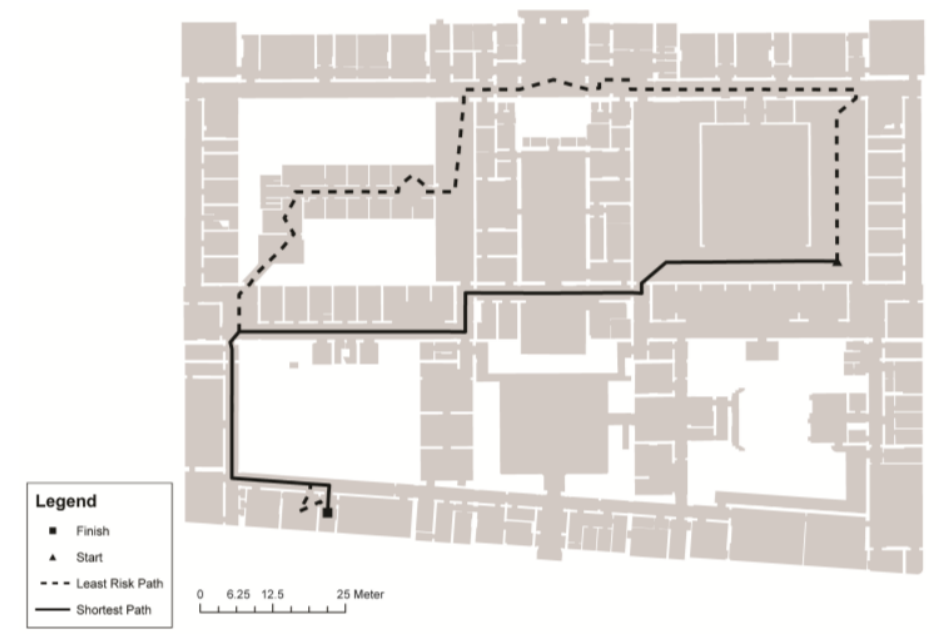

Fig. 7: Comparison of a typical shortest and least risk path (floor 0)

Table 7 enumerates on the parameters used in the algorithm itself (first 3 lines) and the selected benchmark parameters. For the parameters used in the algorithm itself, the results are as expected: a lower total risk value for the least risk path with a considerable lower risk value at the individual decision points, by choosing a longer route (43\% longer in this case). The other parameters, however, show a different side of the coin, with better results for the shortest path 
algorithm in terms of reducing the risk of getting lost. For example, the shortest path has 7 turns in its description, while the least risk path requires 12 turns. Wayfinding literature has extensively shown that more turns considerably increase the risk of disorientation and as such also the risk of getting lost by taking wrong decisions. The chosen corridors in the least risk path algorithm are generally less integrated, with less visibility towards the next decision points (4.68 versus 5.17) and a higher route complexity (more decision nodes passed on the total route, more curves and more spatial units passed). Above result indicates a less comfortable (and much longer) route traversing for unfamiliar users compared to the shortest path. In this case, the least risk path algorithm performs worse in terms of choosing less risky edges which completely undermines the initial intentions of the algorithm. The suggested shortest path will probably be closer to the natural wayfinding behavior of unfamiliar users compared to the least risk path algorithm. Together these examples demonstrate that even though an accurate route is often proposed by the least risk path algorithm, just as often a more risky and uncomfortable route is suggested.

Table 7: Comparison of the parameters between an example shortest and least risk path

\begin{tabular}{lcc}
\hline Benchmark parameters & Shortest paths & Least risk paths \\
\hline Risk values of decision points (average; $m)$ & 274.27 & 166.36 \\
Risk value of the entire path $(m)$ & 445.07 & 411.79 \\
Total path length & 170.80 & 245.43 \\
\hdashline No. of turns & 7 & 12 \\
No. of spatial units passed & 6 & 3 \\
No. of curves & 0 & 3.2 and 2 \\
Width of corridors (m) & 3.2 & 37 \\
No. of decision nodes passed & 29 & 4.68 \\
No. of visible decision nodes at each decision node (average) & 5.17 & \\
\hline
\end{tabular}

\section{Discussion}

\subsection{Summary of the results of the case study}

The case study revealed some interesting results with regard to the applicability of the least risk path algorithm in indoor spaces. First, it was shown that on average least risk paths are only $4 \%$ longer than their respective shortest paths, with $47 \%$ of the entire dataset having equal path lengths and as such equal spatial embedding. Also, from the paths deviating from their shortest path equivalent, $98 \%$ has a limited deviation (less than $25 \%$ longer path length) of only here and there a different side route and this mostly through rooms avoiding main corridor areas. Second, the navigational complexity analysis showed again similar results over both algorithms, but the least risk paths were often longer with a similar path complexity. If the least risk path algorithm decides to deviate from the shortest path alternative, it should be supported by taking less risky and complex routes, which is not the case. Third, for paths with a significantly different path embedding, the least risk path ended up sometimes less risky when compared to our benchmark parameter set, but evenly as many times the shortest path would be preferred as least risky.

This leads to the main conclusion that the least risk path algorithm does not return stable results in terms of selecting the least risky edges in indoor environments. For short path lengths the 
similarity between both algorithms in terms of path embedding seems reasonable as the density of the indoor network (and the importance of staircases in the indoor graph) impedes many deviations. However, on longer total path lengths, deviations have been noticeable, sometimes for the better, but evenly as many times it resulted in taking theoretically more risky and cognitively more difficult routes. Also, the deviations from the main corridor to side rooms are running counter to typical wayfinding strategies. Therefore, we are inclined to say that at this point for indoor wayfinding, the least risk path algorithm calculates alternative routes between two points, without necessarily reducing navigational complexity. This leads us to believe that the least risk path algorithm and its definition of risk should be investigated in more detail and altered to be more aligned to the specificities of indoor wayfinding. In the following section, we will discuss several reasons for this misalignment between algorithm and the specific indoor situation and afterwards propose some improvements to the original algorithm.

\subsection{Reasons for misalignment}

\subsubsection{Risk value definition}

The minimization criterion of least risk is composed of a path length value and a risk value. For most algorithms, the total path length plays in some way a vital role in determining which edges get selected. The introduction of the risk value is specific to this algorithm and could be one of the reasons for the current inaccurate results. At this point, the risk value takes into account the number of streets converging at an intersection and their individual lengths, to obtain a kind of average length of a wrong edge at that intersection. In the following paragraphs, the implications of defining the risk value in this way are examined in more detail.

First, the individual lengths of the wrong segments are key in the calculation of the intersectionbased risk value. By only utilizing the length of wrong edges, the algorithm will initially always select the edge with the longest individual path length, as this edge would add the most to the average wrong path length if not selected. The more equal all edges at an intersection are, the more similar the risk values will be. During the entire run of the algorithm, a more balanced optimum will be created over time were sometimes edges are selected with a slightly lower risk value. However, during the actual wayfinding act the individual lengths and length ratios between all edges at an intersection is not necessarily of importance in having more or less chances of getting lost during the trajectory. Selecting as many possible long edges is important (theoretically less intersections over the total path length), as long as this not results in bumping into really complex or confusing intersections. The algorithm actually does provide this selection of long edges in its current form. However, selecting an edge with a slightly shorter length but with other parameters that reduce navigational complexity (e.g. a long line of sight, wide and open corridor ...) might often be more important for overall risk reducing than just the length of the edge in relation to the other edges alone.

The second parameter in the formula of the risk value calculation is the number of choices at an intersection. This parameter aims to cover the effect of the intersection's complexity (i.e. the amount of edges converging at an intersection) on the risk of getting lost. The analyses in section 3.2.2 have shown that the average number of choices at an intersection in the least risk path algorithm is fairly similar to the results of the shortest path algorithm. This implies that this parameter in the calculation of the risk value does not necessarily add much to the final risk value. Fig. 8 plots the relationship between intersection complexity and risk value. It shows an exponential relationship where with increasing intersection complexity the risk value increases 
with relatively smaller amounts. This demonstrates that the amount of edges converging actually does have an importance on the risk value. However, after a certain point, the relative importance of adding more choices at an intersection does not really have a significant effect on the final risk value. Even though having a slight increase of possible choices at an intersection might not add much more discomfort for the wayfinder itself, his chance of picking the right option does actually decrease.

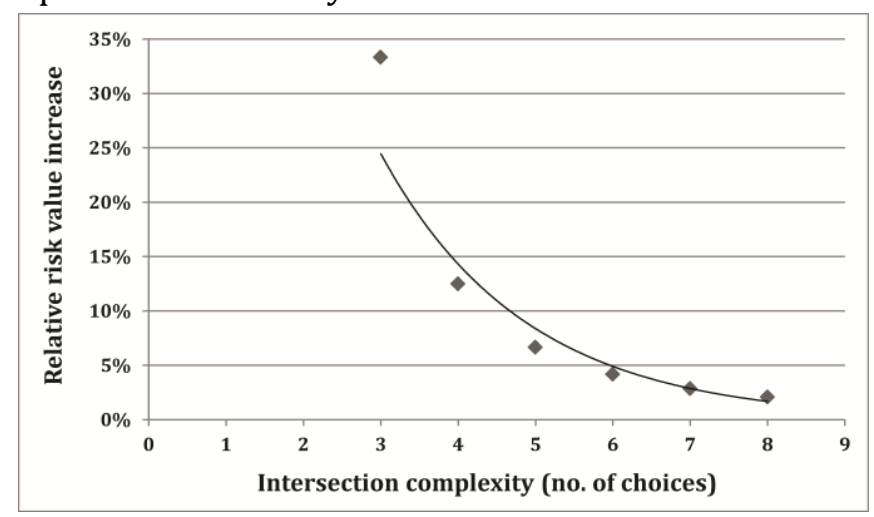

Fig. 8: Relationship between intersection complexity and relative risk value increase

In conclusion, some aspects in the risk value calculation do seem to make sense helping people avoid getting lost and choosing more optimal paths. However, the importance of the intersection complexity is not as profound as might actually be necessary in wayfinding. At this point, the selection of the longest possible edge gains the upper hand over the intersection complexity. This might indicate a possible reason for the wrongful selecting of less risky paths and requires adjustment of the original definition of risk value.

\subsubsection{Network definition}

At this point, the least risk path algorithm indoor was tested using a Geometric Network structure as defined by Lee (2004). Apart from representing each spatial unit by a single node, the key element of this network structure is the transformation of corridor-labelled units to linear features. As described in Lee (2007, p. 516) "the 3D GNM is a topological data model representing the connectivity relationships among discrete objects and the geometric properties of objects in three-dimensional space (e.g., location in 3D space, distance between two rooms, and length of a hallway)". The transformation of corridors into a sub network consolidate hallway nodes in the combinatorial network by projecting and connecting door way points onto the medial axis of the corridor (Lee, 2004). The goal of this transformation is to upgrade a solely topological model of connectivity relationships into a geometric network model representing more accurately paths of movement between all units. As an effect, each corridor is often subdivided in many nodes in front of each doorway interconnected by short edges (Fig. 9).


Fig. 9: Topologic connectivity network (left) versus Geometric network (right)

This particular subdivision creates unrealistic results in our calculations of least risk paths. With the creation of these synthetic hallway intersections, more intersections have to be possibly passed, with each intersection adding more weight to the total risk value of the path. Also, as 
discussed previously, the original algorithm selects the longest edge in each intersection in its risk calculation. Fig. 10 shows that this can lead to deviations of the final least risk path from the main corridor as the longest edge in the intersection leads towards a room on the side having two connecting doors to the corridor. This was also confirmed in section 3.3.1 with deviations in the least risk paths being mostly through room areas instead of corridors. On top of that, this example also demonstrates that avoiding the short edges of the main corridor leads to a lower total risk value as the node in the selected room does not cause the calculation of an additional risk value (the node has only two edges converging). The exact examination of the influence of this particular network type on our results of the least risk path algorithm is subject for future work.

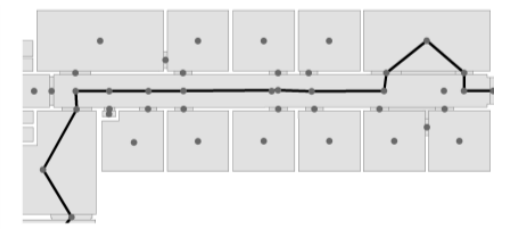

Fig. 10: Zoom of the example from path selection in Fig. 7

This example shows an unrealistic walking pattern as there is no apparent reason in the eyes of the wayfinder for this deviation from the straight corridor line. Also, knowing exactly which room to enter is more complicated in this case with many doors and rooms on both sides of the corridor inducing more options and choices to be made. This illustrates an additional problem with this type of network. When having to traverse an entire corridor, the synthetic hallway nodes are often not perceived as intersections or decision points by the user. This was also proven in wayfinding experiments where participants explicitly stated not requiring any landmark checkpoints in a corridor, as only new information was needed when choices had to be made about the remainder of the route (Viaene \& De Maeyer., 2013). It also underlines the difference between outdoor urban networks and the indoor equivalent: in outdoor space each intersection represents a formal decision point, while this is not necessarily the case in indoor environments. This is especially true when traversing a corridor with only closed doors (often in office buildings) leading to private rooms, while the unfamiliar user might only have access to the publically traversable corridor.

\subsubsection{Indoor versus outdoor space differences}

Indoor and outdoor spaces are considerably distinct in structure, constraints and usage. Although both environments are often consisting of linear structures with obstructions, the human perception during navigation is entirely different. Outdoor urban environments have mostly a wider view with no covering which is sensed as uncluttered and orderly space, even in dense city environments. Indoor environments have often more discontinuities and are totally covered, which is perceived as a fragmented, enclosed and clustered environment (Richter et al., 2011). This difference in human perception has to seep through in the algorithmic support as it highly influences the risk of getting lost. This also demonstrates why the risk value for indoor application might require a more complex and coherent approach compared to outdoor spaces.

The transformation into an appropriate network has shown to create some additional problems for application of the algorithm. This has its origin in the different network complexity of both spaces. Most buildings contain several major corridors with rooms on the side containing only one exit, while outdoor street networks are in general more integrated leaving several options for path alternatives. This also explains the high similarity in results between least risk and shortest paths in our indoor tests. There are often not many options to deviate from the shortest 
path, making the deviations that occur being more important to provide users in an easier navigation experience.

\subsection{Possible improvements to the algorithm}

\subsubsection{Weight adjustment}

Several options for adjusting the internal weight balance are possible in the algorithm. The most straightforward one is altering the relevance given to the parameters in the current algorithm. In the original implementation of the least risk path algorithm, both the length of the path as well as the sum of the risk values at intermediate decision points add an equal weight in the calculation of the overall risk value. Changing this ratio of length versus risk value might result in a more cognitively correct selection of least risk paths indoor. To examine this, the original definition can be improved by adding two parameters $\alpha$ and $\beta$, one for each variable, with their mutual sum always equal to 1 .

Total_Risk $(p)=\propto *$ PathLengths $+\beta *$ RiskValue $(i)$

$\alpha+\beta=1$

As an example of this process, the weights of the path presented in Fig. 6 are altered with the results visualized in Fig. 11.



Fig. 11: Weight adjustment by changing the mutual importance of risk value versus path length

The orange line $(\alpha=0.5)$ visualizes the original least risk path with equal importance to path length and risk value. Changing the importance of the length to a lesser amount apparently does not change much in the final path choice in this example. Only an additional deviation through non-corridor areas $(\alpha=0.4)$ is included as a result of the added importance to the risk value calculations, which leads to an even higher avoidance of short edges and intersections. From $\alpha=0.6$, the route starts to coincide more with the shortest path $(\alpha=1)$. However, the route deviates to an outdoor courtyard area to later on join the original shortest path again. Even though in both cases the path traverses main corridors and outdoor areas, an unfamiliar user would probably prefer the shortest route as its least risk path, as it does not require any physical changes of spatial unit in contrast with the least risk path $(\alpha=0.6)$ (physically going outside using two small doors). This extra attribute might also need to be added to the network. Note that in 
case of $\alpha=0.7$ the path deviates once more from the main corridor due to the definition of both network and risk value. In this case, given the high weight to path length in favor of risk value, the network structure will be the defining variable. A more hierarchical network structure is thus highly recommended. This is only an example showing the possibilities of altering the mutual relationship of the main parameters defining the total risk value. At this point we cannot give any further indication on the best ratio of $\alpha$ and $\beta$ parameters as it requires comparisons between multiple start- and endpoints and even in buildings with a different spatial structure.

A second possibility of weight adjustments exists in changing the internal definition of risk value by adding more parameters relevant to minimizing the risk of getting lost during wayfinding. In section 4.2.1, it was already proven that the current definition of risk value is rather limited with the selection of the longest possible edge gaining the upper hand over the intersection complexity. In Table 2, several other factors were listed as theoretically important in optimizing wayfinding situations. The individual weighting of these parameters is up for future research. However, we would like to propose a division of the current risk value into an intersection based risk value and an edge based risk value (Equation 5).

$$
\text { Total }_{\text {Risk }(p)}=\propto * \text { PathLengths }+\beta * \text { RiskValue }(i)+\gamma * \text { RiskValue }(\text { edge })
$$

The risk value of selected edges is of importance since at this point no aspects denoting the overall individual importance of each edge apart from the edge length (e.g. width, number of curves, integration value) are yet incorporated in the assessment of risk. These variables are tightly linked to the edge structure and completely independent of the intersections themselves. On intersection level, other aspects that can influence the edge choice for continuation of the path, like the directional orientation of each edge at the intersection, are also not yet considered. The intersection-based risk value can also be influenced by the same parameters denoting the individual importance of the edge, but on a more local level. For example, the sight of several small corridors and a single large corridor at an intersection will highly influence path choice and comfort when selecting the widest corridor and not the smallest variant. Experiments with defining various risk value definition with more parameters from Table 2, individually weighted, are considered as future work.

\subsubsection{Other possible algorithmic improvements}

In this final section, we will suggest some other improvements to the original algorithm which will be tested and compared in our future research.

First, the risk value of a decision point is currently calculated based on the assumption that the wayfinder recognizes his mistake at the first adjacent node and returns from there to the previous node. The question could be raised whether it is actually realistic that people already notice at the first intersection that they have been going wrong. An increasing compounding function could be suggested taking into account the possibility of going further in the wrong direction.

Second, given the importance of an appropriate network topology, a more sophisticated algorithm could select routes that preferentially use more important or higher classified edges to be in line with users hierarchical spatial reasoning. The main question here is which hierarchical structure should be used and how should it be defined. In outdoor navigational research, the road classification often serves as natural hierarchy. However, this hierarchy is 
much harder to define for indoor spaces. A possibility could be to discover the latent natural hierarchy of the indoor graph by using the reach metric introduced by Gutman (2004).

Related to this topic is the importance of staircases, as it was proven that they are key elements in the indoor path selection. The fact that you have to walk up and down staircases during a certain route could be naturally having a greater weight because taking a wrong decision might result in walking up and down the stairs twice. On the other hand, chances of making a wrong decision by changing floors are likely to be slimmer given the effort required for vertical movement. Additionally, it has been found that the number of rotations on a staircase plays a major role in keeping stability in the user's cognitive map. Hölscher et al. (2012) identified many getting-lost episodes due to disorientation after leaving a staircase, sometimes even on the wrong floor.

Fourth, Hölscher et al.'s (2009) wayfinding research has proven that people's strategy choice indoors varies with different navigation tasks. Tasks with either a floor change or a building part change result in no problems, with the participants first changing to the correct floor or building part. However, for tasks with changes in both vertical and horizontal direction, additional information is required to disambiguate the path choice. An algorithm that wants to minimize the risk of getting lost in a building necessarily needs to account for these general indoor wayfinding strategies as they correspond to the natural way of multilevel building navigation for all types of participants.

\section{Conclusions}

In this paper, the least risk path algorithm as developed by Grum (2005) in outdoor space was implemented and tested in an indoor environment to examine its suitability for indoor wayfinding. The results of those tests have shown that with a slight increase in path length, theoretically less risky paths were calculated. However, further analyses have demonstrated that these least risk paths are not necessarily significantly different, nor are they optimal in terms of reducing navigational complexity and getting-lost episodes. This leads to the conclusion that a dissonance exists between the original definition of the algorithm and its implementation in indoor environments. Several suggestions were made to improve the algorithm, ranging from changes in the calculation of the risk value, to individual selection and weighting of the parameters involved, to the influence of the indoor network topology. The aim for future research is to discover the best optimization of the algorithm to make it more compliant with the cognitive notion of indoor wayfinding. More generally, this research will aid the development of appropriate tools that improve navigation experiences in indoor spaces.

\section{Acknowledgments}

Financial support from the Flanders Research Foundation (FWO-Vlaanderen) is gratefully acknowledged. The use of the building CAD files was kindly permitted by the Department of Infrastructure and Facility Management of Ghent University. 


\section{References}

Anagnostopoulos, C., Tsetsos, V., Kikiras, P., \& Hadjiefthymiades, S. P. (2005). OntoNav: A Semantic Indoor Navigation System. 1st Workshop on Semantics in Mobile Environments (p. 8). Ayia Napa, Cyprus.

Atila, U., Karas, I., \& Rahman, A. (2013). A 3D-GIS Implementation for Realizing 3D Network Analysis and Routing Simulation for Evacuation Purpose. In J. Pouliot, S. Daniel, F. Hubert \& A. Zamyadi (Eds.), Progress and New Trends in 3D Geoinformation Sciences pp. 249-260. Springer Heidelberg.

Becker, T., Nagel, C., \& Kolbe, T. (2009). A Multilayered Space-Event Model for Navigation in Indoor Spaces. In J. Lee \& S. Zlatanova (Eds.), 3D Geo-Information Sciences pp. 61-77. Springer Heidelberg.

Brown, G., Nagel, C., Zlatanova, S., \& Kolbe, T. (2013). Modelling 3D Topographic Space Against Indoor Navigation Requirements. In J. Pouliot, S. Daniel, F. Hubert \& A. Zamyadi (Eds.), Progress and New Trends in 3D Geoinformation Sciences pp. 1-22. Springer Heidelberg.

Carlson, L. A., Hölscher, C., Shipley, T. F., \& Dalton, R. C. (2010). Getting lost in buildings. Current Directions in Psychological Science, 19, 284-289.

Dijkstra, E. W. (1959). A Note on Two Problems in Connexion with Graphs. Numerische Mathematik, 1, 269-271.

Duckham, M., \& Kulik, L. (2003). "Simplest" Paths: Automated Route Selection for Navigation. In W. Kuhn, M. Worboys \& S. Timpf (Eds.), Spatial Information Theory. Foundations of Geographic Information Science pp. 169-185. Springer Heidelberg.

Fu, L., Sun, D., \& Rilett, L. R. (2006). Heuristic shortest path algorithms for transportation applications: State of the art. Computers \& Operations Research, 33, 3324-3343.

Golledge, R. G. (1999). Human Wayfinding and Cognitive Maps. In R. G. Golledge (Eds), Wayfinding Behavior: Cognitive Mapping and Other Spatial Processes pp. 5-45. The Johns Hopkins University Press Baltimore.

Grum, E. (2005). Danger of getting lost: Optimize a path to minimize risk. 10th International Conference on Information \& Communciation Technologies (ICT) in Urban Planning and Spatial Development and Impacts of ICT on Physcial Space pp. 711-717. Vienna, Austria: CORP 2005.

Gutman, R. (2004). Reach-based Routing: A New Approach to Shortest Path Algorithms Optimized for Road Networks. 6th International Workshop on Algorithm Engineering and Experiments pp. 100-111.

Haq, S., \& Zimring, C. (2003). Just Down The Road A Piece: The Development of Topological Knowledge of Building Layouts. Environment and Behavior, 35, 132-160.

Hölscher, C., Brösamle, M., \& Vrachliotis, G. (2012). Challenges in multilevel wayfinding: a case study with the space syntax technique. Environment and Planning B: Planning and Design, 39, 63-82. 
Hölscher, C., Büchner, S. J., Meilinger, T., \& Strube, G. (2009). Adaptivity of wayfinding strategies in a multi-building ensemble: The effects of spatial structure, task requirements, and metric information. Journal of Environmental Psychology, 29, 208-219.

Hölscher, C., Meilinger, T., Vrachliotis, G., Brösamle, M., \& Knauff, M. (2006). Up the down staircase: Wayfinding strategies in multi-level buildings. Journal of Environmental Psychology, 26, 284-299.

Hölscher, C., Tenbrink, T., \& Wiener, J. M. (2011). Would you follow your own route description? Cognitive strategies in urban route planning. Cognition, 121, 228-247.

Kwan, M.-P., \& Lee, J. (2005). Emergency response after 9/11: the potential of real-time 3D GIS for quick emergency response in micro-spatial environments. Computers, Environment and Urban Systems, 29, 93-113.

Lee, J. (2004). A Spatial Access-Oriented Implementation of a 3-D GIS Topological Data Model for Urban Entities. Geoinformatica, 8, 237-264.

Lee, J. (2007). A Three-Dimensional Navigable Data Model to Support Emergency Response in Microspatial Built-Environments. Annals of the Association of American Geographers, 97, 512529.

Mark, D. M. (1986). Automated route selection for navigation. IEEE Aerospace and Electronics Systems Magazine, 1, 2-55.

Meijers, M., Zlatanova, S., \& Pfeifer, N. (2005). 3D Geo-Information Indoors: Structuring for Evacuation. Next generation 3D City Models (p. 6). Bonn, Germany.

Montello, D. R. (2005). Navigation. In P. Shah \& A. Miyake (Eds.), The Cambridge Handbook of visuospatial thinking (pp. 257-294). Cambridge University Press New York.

Musliman, I. A., Abdul-Rahman, A., \& Coors, V. (2008). Implementing 3D network analysis in 3DGIS. 21st ISPRS Congress Silk Road for Information from Imagery pp. 913-918. Beijing, China: International Society for Photogrammetry and Remote Sensing.

Nagel, C., Becker, T., Kaden, R., Li, K.-J., Lee, J., \& Kolbe, T. H. (2010). Requirements and SpaceEvent Modeling for Indoor Navigation. OGC Discussion Paper (p. 54). OGC.

Open Geospatial Consortium (2013). OGC IndoorGML. (p. 72). Lee J., Li K.J., Zlatanova S., Kolbe T.H., Nagel C., Becker T.

Parvin, A., Ye, A. M., \& Jia, B. (2007). Multilevel pedestrian movement: does visibility make any difference? 6th International Space Syntax Symposium pp. 40.01-40.16. Istanbul, Turkey.

Peponis, J., Zimring, C., \& Choi, Y. K. (1990). Finding the Building in Wayfinding. Environment and Behavior, 22, 555-590.

Richter, K.-F., \& Duckham, M. (2008). Simplest Instructions: Finding Easy-to-Describe Routes for Navigation. In T. Cova, H. Miller, K. Beard, A. Frank \& M. Goodchild (Eds.), Geographic Information Science pp. 274-289. Springer Heidelberg. 
Richter, K. F., Winter, S., \& Santosa, S. (2011). Hierarchical representations of indoor spaces. Environment and planning B, Planning and design, 38, 1052-1070.

Stoffel, E. P., Schoder, K., \& Ohlbach, H. J. (2008). Applying Hierarchical Graphs to Pedestrian Indoor Navigation. Proceedings of the 16th ACM SIGSPATIAL international conference on Advances in geographic information systems. Irvine, California: ACM.

Thill, J.-C., Dao, T. H. D., \& Zhou, Y. (2011). Traveling in the Three-Dimensional City: Applications in Route Planning, Accessibility Assessment, Location Analysis and Beyond. Journal of Transport Geography, 19, 405-421.

Tsetsos, V., Anagnostopoulos, C., Kikiras, P., \& Hadjiefthymiades, S. (2006). Semantically enriched navigation for indoor environments. International Journal of Web and Grid Services, 2(4), 453-478.

Turner, A., Doxa, M., O'Sullivan, D., \& Penn, A. (2001). From isovists to visibility graphs: a methodology for the analysis of architectural space. Environment and planning B, Planning and design, 18(1), 103-121.

Vanclooster, A., Neutens, T., Fack, V., Van de Weghe, N., \& De Maeyer, P. (2012). Measuring the exitability of buildings: A new perspective on indoor accessibility. Applied Geography, 34, 507518.

Viaene, P., \& De Maeyer, P. (2013). Detecting landmarks for use in indoor wayfinding. Master thesis, Department of Geography, Ghent University, Ghent. 